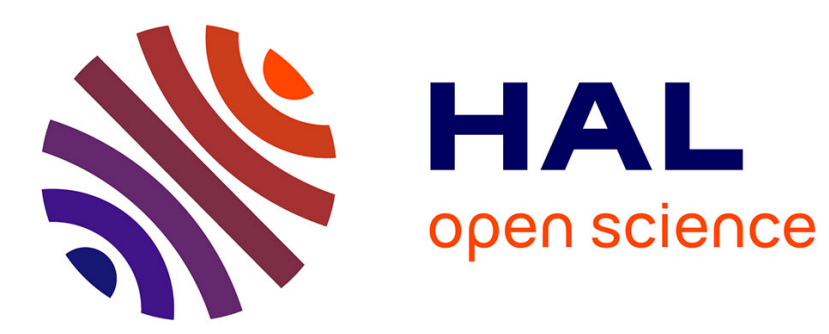

\title{
Dynamic structure identification of Bayesian network model for fault diagnosis of FMS
}

\author{
Dang-Trinh Nguyen, Quoc Bao Duong, Éric Zamaï, Muhammad Kashif \\ Shahzad
}

\section{- To cite this version:}

Dang-Trinh Nguyen, Quoc Bao Duong, Éric Zamaï, Muhammad Kashif Shahzad. Dynamic structure identification of Bayesian network model for fault diagnosis of FMS. The 40th Annual Conference of the IEEE Industrial Electronics Society, Oct 2014, Dallas, Texas, United States. pp.7. hal-01058756

\section{HAL Id: hal-01058756 https://hal.science/hal-01058756}

Submitted on 28 Aug 2014

HAL is a multi-disciplinary open access archive for the deposit and dissemination of scientific research documents, whether they are published or not. The documents may come from teaching and research institutions in France or abroad, or from public or private research centers.
L'archive ouverte pluridisciplinaire HAL, est destinée au dépôt et à la diffusion de documents scientifiques de niveau recherche, publiés ou non, émanant des établissements d'enseignement et de recherche français ou étrangers, des laboratoires publics ou privés. 


\title{
Dynamic structure identification of Bayesian network model for fault diagnosis of FMS
}

\author{
Dang Trinh Nguyen*, Quoc Bao Duong ${ }^{\dagger}$, Eric Zamai*, Muhammad Kashif Shahzad* \\ ${ }^{*}$ Grenoble-INP, G-SCOP Laboratory \\ 46 avenue Felix Viallet, 38031 Grenoble Cedex, France \\ $\dagger$ A.I.P PRIMECA Dauphine Savoie \\ 740 Rue de la piscine BP 46, 38402 ST Martin D'Heres Cedex, France \\ \{Dang-Trinh.Nguyen, Quoc-Bao.Duong, Eric.Zamai, Muhammad-Kashif.Shahzad\}@grenoble-inp.fr
}

\begin{abstract}
This paper proposes an approach to accurately localize the origin of product quality drifts, in a flexible manufacturing system (FMS). The logical diagnosis model is used to reduce the search space of suspected equipment in the production flow; however, it does not help in accurately localizing the faulty equipment. In the proposed approach, we model this reduced search space as a Bayesian network that uses historical data to compute conditional probabilities for each suspected equipment. This approach helps in making accurate decisions on localizing the cause for product quality drifts as either one of the equipment in production flow or product itself.
\end{abstract}

Index terms-Fault diagnosis, Flexible Manufacturing Systems, Logical diagnosis, Bayesian network.

\section{INTRODUCTION}

The manufacturing processes evolve to adapt with the increasing demand diversities. This has resulted in complex manufacturing environment where it is difficult to control, diagnose and recover the abnormal events in timely manner. The fault detection and diagnosis techniques have been a special focus in the manufacturing industries to improve product quality and reduce associated costs. Therefore, different methods are developed for more accurate and quick fault diagnosis to improve product quality such as [1], [2]. An advantage of these methods is the flexibility to adapt to different production systems.

However, modern production systems comprise a lot of equipment that are monitored by thousands of sensors. This result in huge amount of information and makes it difficult to identify the model for fault diagnosis with corresponding variables. The artificial intelligence methods are practical techniques capable of using large amount of information from large production databases as presented in [3], [4]. These approaches can be performed without understanding the underlying structure of a production system [5]. Especially, the Bayesian network (BN) approaches are widely used to compute the conditional probabilities between a detected fault and a set of potential failure candidates (root causes). This set and these relations between variables are normally modeled through a graphical structure during learning phase of $\mathrm{BN}$. The associated probabilities help to make accurate and informed decisions about corrective maintenance. However, due to the complexity of present-day manufacturing systems, this graphical structure identification is a complex task to be performed by a personal operator [5]. In addition, it also depends on the database size and the experts knowledge [6]. Besides, the diversity of system characteristics (structure, product type, recipe and the human factor) and the dynamic feature of production systems add to the difficulty in BN structure identification. In fact, the system elements are frequently changed due to the introduction of new manufacturing technologies and management philosophies. Due to a change in one element may trigger effect to others, we must retrain the learning step of a BN model to adapt with newly emerging situations in a production system. In this case, the time and work-load for control system are very large.

In summary, the paper focuses on an approach to dynamically generate the structure of $\mathrm{BN}$ and associated probabilities from the reduced search space of suspected production equipment as identified by the logical diagnosis model. For this context, we propose the use of logical diagnosis model [2] to limit the space of root causes and provide the cause-consequence relationships for the graphical structure identification. Thus, it is followed by learning conditional probabilities in resulting $\mathrm{BN}$ to accurately identify the cause of product quality drift as either a suspected equipment in the production flow or product itself. This approach enables real time diagnosis and subsequent corrective maintenance in a flexible manufacturing system (FMS).

The next section will introduce the characteristic of the case study and objectives for real time diagnosis in FMS. Section 3 presents state of the art fault diagnosis models being used in complex manufacturing environments. The proposed diagnosis presented and discussed in Section 4. The proof of concept is presented in Section 5. We conclude this paper with conclusions and future perspectives in Section 6.

\section{Flexible MANufacturing System}

In the manufacturing industry, speed, reliability, flexibility, cost, rapid product innovation and quality are all related to design a manufacturing process [7]. Many types of manufacturing system as presented in [8] support the design and management of manufacturing processes. Following the variety of the customer demands, new products frequently are introduced. This requires FMS [9] that is widely used in large scale production industries like semiconductor and automotive industries. A FMS is a complex automated production system which consists of several production stations each specializing in particular operations with an appropriate transport system. 


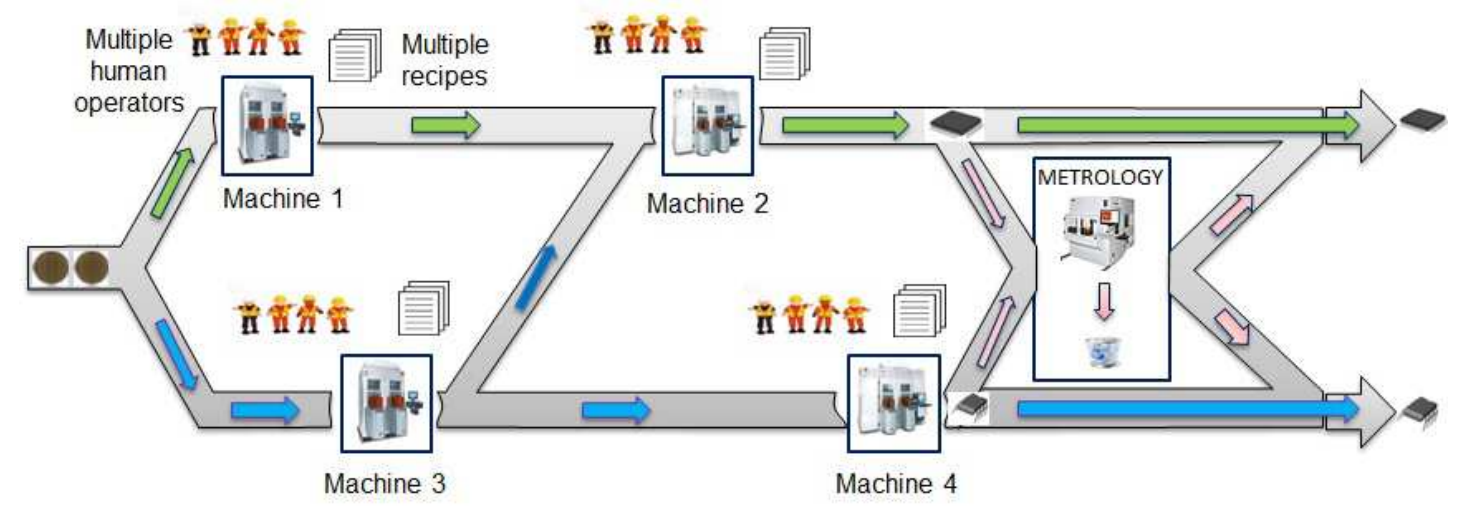

Fig. 1. A Flexible Manufacturing System

These production stations and transport system (controlled system) are controlled by a control system. The computer system coordinates the activities and executes the demands based on control laws. The flexibility of FMS is generally demonstrated by multiple products, multiple lines, multiple recipes, and multiple human factors. Generally, a complex system may have many production processes. The production process is loaded corresponding to each product and executed by the control system. The structure of a production process describes type of product, product lines and corresponding equipment in operating part of controlled system. Any change in the existing product or product line, requires customization of existing process.

Our research is focused on the general framework of a complex system with dynamic production environments. This case study has the characteristics: multiple products, multiple lines, multiple recipes, and multiple human factors as illustrated in Fig.1.

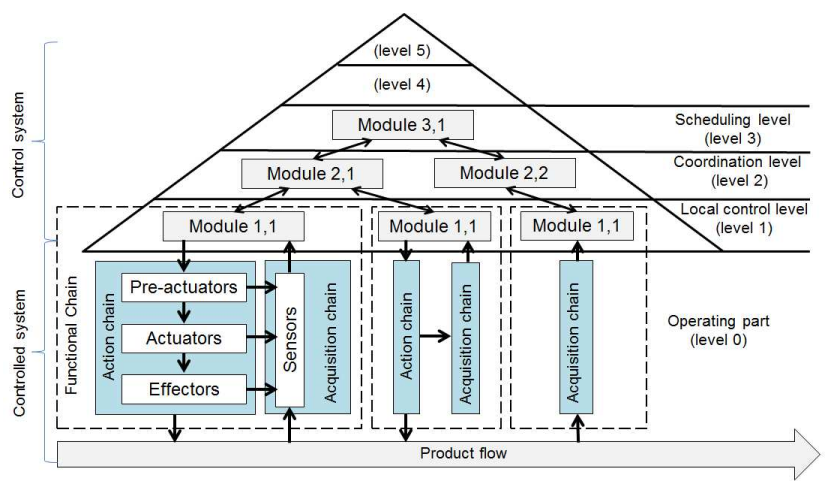

Fig. 2. CIM architecture of an automated manufacturing system

In complex engineering applications, systems are composed of many components and subsystems, and the ways in which these elements interact will affect the way failures propagate within subsystems and across subsystem boundaries [10]. For monitoring the execution of these elements, the hierarchical and modular control are often used as presented in [11]. In this context, an automated manufacturing system is organized by Computer Integrated Manufacturing (CIM) architecture that contains : controlled system, product flow and control system as shown in Fig.2. The controlled system con- sists of actuators and sensors. These sensors allow controlling and monitoring the executions of actuators and product flows. Each elementary part of the controlled system (actuators and sensors) is controlled by a local control module, and these two elements are called Functional Chain (FC) [12]. These FCs receive and execute demands from the coordination level of control system. When a FC cannot correctly execute a demand, it implies that a fault is produced. A fault may be created by a component and can propagate to other components through product lines. Therefore, this failure propagation may make consequences on many components; and so, the new faults may continue to occur. Thus, when a fault is detected by a metrology, its root causes may come from one or a part of elements in the production system. In short, it is critical to precisely and quickly locate the causes of detected fault to save recovery time (for return to a normal status) of the production system.

\section{GENERAL SITUATION}

For fault diagnosis in a production equipment or a simple system, the diagnosis methods such as Fault Detection and Isolation (FDI) [13], and Failure Mode and Effects Analysis (FMEA) [14] are classically used. An advantage of these methods is their simple integration in the production system. These approaches generally model all possible failures in a production system using detection information and failure symptoms, collected through sensors. However, due to the complexity of a manufacturing system, it is too difficult to describe all the failures and so their causes. When an unpredicted fault occurs, the diagnosis cannot provide a result without expert support. In fact, a fault can propagate from an equipment to others equipment through product flows and it may have the consequences to others system elements. The unpredicted failures occur quite often; hence, the existing failure model cannot explain such events.

Following failure propagation analysis, diagnosis approaches have been proposed such as propagation graph [15] and temporal chronic [16]. These approaches are based on historical production data to locate operations at the possible origins of detected fault. However, the problems of these approaches are demonstrated as follow: First, the actions of system operations in [15] are considered as independent. In fact, several operations may use the same functional chain to 
execute the demands of control system. Indeed, the corresponding actions are not independent. Second, these approaches do not analyze the behaviors of the FCs, so they cannot explain the consequences between elements of a FC and between different FCs. And finally, these approaches cannot reduce the size of the model. Due to the cyclical operation of the control system, a large amount of information of this production system provokes the problem of combinatory explosion.

Consequently, we are especially interested in the logical diagnosis model proposed in [2]. In this model, a diagnosis function is proposed to characterize historical production data of a controlled system to search suspected origins against the detected fault, in real time. Thus, this model provides a set of possible origins, and its size is reduced by analyzing sensors signals from the controlled system. This is appropriate to diagnose the faults of production equipment and products. However, this model does not show suspect level of each member in this set of fault origins. It is difficult in deciding a maintenance order. In a complex system, the set of possible fault origins is still large and this method would be extended to optimize corrective maintenance activities.

To evaluate the suspect level of fault origins, the probabilistic approaches are widely used such as Neural network [17] and Bayesian network [3]. These probabilistic approaches have capacity to provide the probability values from the large database and the number of corresponding variables of a production system. These probability values allow evaluating the suspect level (high or low) in order to support decision of a maintenance strategy. In particular, the BN models have the advantages which fit to be applied in manufacturing industry as explained in [4]. The methods based on $\mathrm{BN}$ are introduced in [5] to make a diagnosis for a multiple variables system. The Confidence level of feedback information is proposed in [18] to provide the probability value which shows the correct actions confidence of reported information of equipment. When the database is available, these approaches are strong tools for the corrective maintenance.

However, the probabilistic approaches must be extended. First, the structure of model for Confidence level of feedback information in [18], is static with seven parameters. In the context of flexible manufacturing system with the characteristics such as multiple products, multiple lines, multiple recipes and multiple human factors, the production environment often change as we presented in above section. Thus, the set of parameters which can have impact on the equipment confidence is dynamic. And second, the $\mathrm{BN}$ model must be rebuilt with new contextual information from the production database. In practice, when the database is available, learning approaches are often used for modelling $\mathrm{BN}$ as presented in [19]. In the learning approaches, a graphical structure and probabilistic rules are estimated from observed data. We can cite a number of learning approaches introduced in [6], [19] such as EM, Maximum likelihood, IC\&IC, K2, and Genetic algorithm. Many studies in [5], [19] show that these learning approaches are still complex in identification the variables for a human agent because they depend strongly on expert opinions. Furthermore, the learning workload for a calculating operator is still large [19]. This spends too much time and is not appropriate to on-line structural identification due to this depends on the exploitation of databases, the experiences of experts and the capacity of a calculating operator. In fact, production environments are increasingly stressed by strong competition. It shows that the time for locating the root causes of failures and process recovery (return the process to a normal status) is very important. These challenges promote the researches to apply $\mathrm{BN}$ model for real time fault diagnosis and corrective maintenance optimization.

In this paper, we propose a diagnosis model that allows real time localization of the possible fault root causes, thus dynamically calculating the conditional probabilities between a fault and its causes. This model is based on a logical diagnosis model and a $\mathrm{BN}$ model. It has four main points: First, the logical diagnosis model provides a set of possible fault origins. The relationships of members in this set are used to construct a graphical structure. Second, we use this graphical structure for the structural modeling of $\mathrm{BN}$ model. This idea is to simplify the variables identification in learning step of the BN. Third, the historic information from the production system is used to estimate probabilistic rules in learning step of $\mathrm{BN}$ model. And finally, the conditional probabilities of nodes in structural model are calculated. These probabilistic values allow evaluating suspect level of each possible fault origin. We will explain in detail this diagnosis model in the next section.

\section{DiAgnOSIS MODEL}

This paper presents a diagnosis model which uses the results given by a logical diagnosis model to dynamically identify the BN structure for fault diagnosis in a flexible manufacturing system. This model comprises a logical diagnosis model and a BN model as shown in Fig.3.

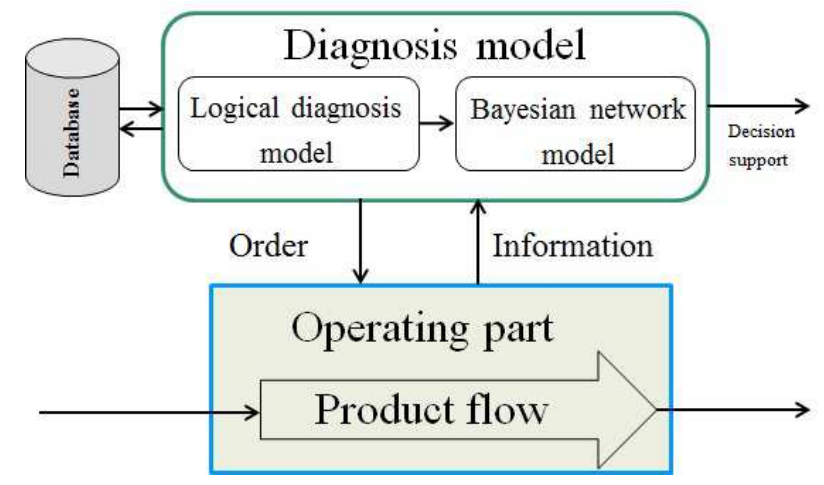

Fig. 3. Diagnosis model

The proposed model consists of: (i) searching possible root causes against a detected fault in the past evolution of the operating part in controlled system, (ii) computing the probability values that show the suspect level against possible root cause. This diagnosis model operates as follows: First, this model is generated by the coordination level of a control system. This coordination level sends commands and receives real time reports for and from all system components. This coordination level also provides the necessary information for the logical diagnosis. Second, once a failure is detected by a metrology, a set of possible origins and its correlations are defined by the logical diagnosis model. Third, the information of this set is sent to BN model. Thus, a graphical structure of failure mode is determined to support the structural identification in learning 
step of BN model. Finally, based on the theories of BN, the corresponding conditional probabilities of all nodes in network are calculated from historical production data. All calculated results are memorized in the database.

The model for diagnosis is based on the control system and dynamic reconfiguration as proposed in [2], [12]. The reactive loop is characterized by collaboration of several supervision, monitoring and control (SM\&C) functions such as detection, diagnosis, prognosis, decision and automatic control [20]. The coordination level of control system has capacity to manage a set of Functional Chains and corresponding information. It also can demand and memorize the information on the database of system. When a fault is detected, this model will demand the diagnosis results from logical diagnosis model and the BN model. We will explain its executions below.

The Logical Diagnosis model proposed in [2] is one part in the treatment of failure propagation through a product in a complex production process. In this model, a diagnosis function is proposed to characterize the historical information of a controlled system in locating the possible origins of a detected fault. This diagnosis function is presented in three main points: First, a model generated real time in normal operation and collects all the necessary information from coordination level for fault diagnosis through the Operation Models which are presented in [12]. An Operation Model consists of information of Functional Chains (equipment, sensors and local control modular), (Pre-)conditions, (Pre-)constraints, effects of operations. This model describes a graphical structure of a production process which consists of system components and its relationships following product flows. Second, a mechanism to reduce this model is developed based on the exploitation of controlled system observations. Following an operation in this model, if the information provided by these two elements is not coherent, the controlled system sends a faulty execution report to the coordination level. If the coordination level does not receive a faulty execution report from these Functional Chains, it can conclude that the corresponding element is reliable. These reliable elements will be removed from the model, while the suspect elements will be retained. And finally, a mechanism is defined, based on the failure propagation approach, that allows us searching for the possible origins and the possible other consequences of a fault when the model receives a faulty execution report. Consequently, this logical diagnosis model provides a set and a reduced structural model of suspect operations which have logical relationships with a faulty execution. These suspect operations are considered as possible origins $\left\{O_{1}, \ldots O_{i}, \ldots O_{n}\right\}$ of detected fault. The reduced model describes the logical links between possible fault origins. The doubt can be propagated from a possible origin to another one. Hence, these logical links are considered as the cause-consequence relationships. This set of possible fault origins and the information of reduced model will be sent to $\mathrm{BN}$ model, and so, they are used for structural identification. Therefore the conditional probabilities are computed next.

The $\mathrm{BN}$ model is based on the $\mathrm{BN}$ theories as presented in [4]. When this model receives a set of possible fault origins and reduced structural model through the coordination level, it will be performed as follows:

- First, an algorithm as presented below is used for equivalent transition from a set of possible root causes and its graphical structure given by the logical diagnosis model to a graphical structure of $\mathrm{BN}$ model. In Algorithm 1, we assume that a set of possible root causes has $n$ members as $\left\{O_{1}, \ldots O_{i}, \ldots O_{n}\right\}$ obtained by logical diagnosis model. Each member $O_{i}$ of the set is considered a node in the $\mathrm{BN}$. The other members $O_{i^{\prime}} ;\left(i^{\prime}=1 \cdots n, i^{\prime} \neq i\right)$ which are in front of the node $O_{i}$ following a product flow and have directly logical relationship with it, are parents of $O_{i}$.

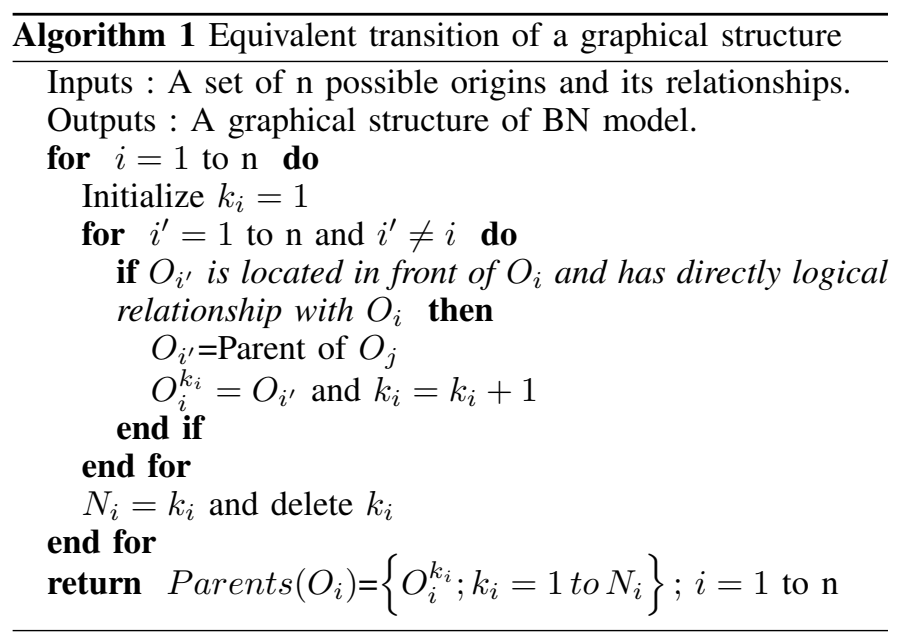

- $\quad$ Second, we obtain a graphical structure of BN model from previous step. Each node in this structure may be a parent of child-nodes and may be a child of other parent-nodes. For instance, a set of parents a detected fault node is a finite number of mutually exclusive operation $\left\{O_{1}, \ldots O_{j}, \ldots O_{N}\right\}$. Each operation $O_{j}$ with $(j=1 \cdots N)$ has a set of parents as $\left\{O_{j}^{1}, \ldots O_{j}^{k_{j}}, \ldots O_{j}^{N_{j}}\right\}$, with $k_{j}=1 \cdots N_{j}$. Therefore, each member $O_{j}^{k_{j}}$ has a set of parents as $\left\{O_{j}^{k_{j}, 1}, \ldots O_{j}^{k_{j}, l_{j}}, \ldots O_{j}^{k_{j}, L_{j}}\right\}$ with $l_{j}=1 \cdots L_{j}$. In this case, the BN model has a hierarchical structure as shown in Fig.4.

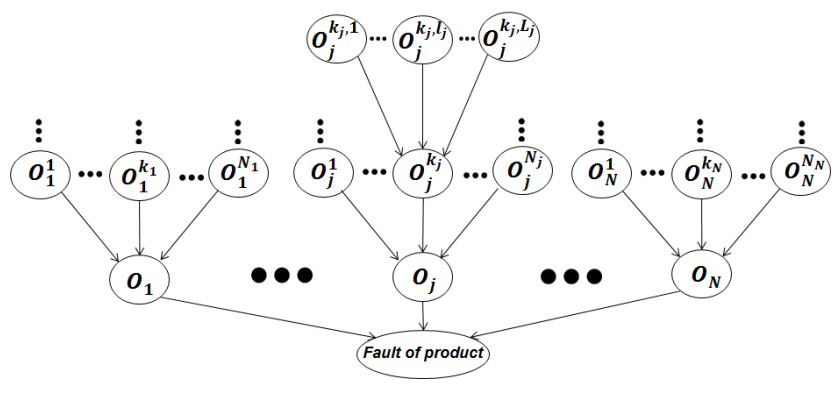

Fig. 4. Graphical structure of BN model

- Third, after a graphical structure is identified, the learning step of $\mathrm{BN}$ model is performed to calculate the probabilities $\mathrm{P}\left(\mathrm{O}_{i} \mid\right.$ Product $)$ of each node $O_{i}$ following the product, and next the conditional probabilities between the child-nodes and its parents based on the historical information of production system 
from the database. We consider that each member of the set of fault origins has two states $\{1,0\}$. So, the conditional probabilities over each operation $O_{j}$ with $(j=1 \cdots N)$ and its parents $\left\{O_{j}^{1}, \ldots O_{j}^{k_{j}}, \ldots O_{j}^{N_{j}}\right\}$ are defined as a matrix $P\left(O_{j} \mid O_{j}^{1}, \ldots O_{j}^{k_{j}}, \ldots O_{j}^{N_{j}}\right)$ as shown in equation (1):

$$
\left(\begin{array}{c}
p\left(O_{j}=1 \mid O_{j}^{1}=1, \ldots O_{j}^{k_{j}}=1, \ldots O_{j}^{N_{j}}=1\right) \\
\vdots \\
p\left(O_{j}=1 \mid O_{j}^{1}=0, \ldots O_{j}^{k_{j}}=0, \ldots O_{j}^{N_{j}}=0\right) \\
p\left(O_{j}=0 \mid O_{j}^{1}=1, \ldots O_{j}^{k_{j}}=1, \ldots O_{j}^{N_{j}}=1\right) \\
\vdots \\
p\left(O_{j}=0 \mid O_{j}^{1}=0, \ldots O_{j}^{k_{j}}=0, \ldots O_{j}^{N_{j}}=0\right)
\end{array}\right)
$$

- In this paper, in order to compute the conditional probabilities, we need to extract the distribution over some subset of variables or a single variable, and thus, we need to marginalize or sum out the variables other than the variables of interest as explained in [21]. The marginalization rule for any sets of variable $\mathrm{X}$ and $\mathrm{Y}$ is given by :

$$
P(X)=\sum_{y} P(X, y) ; y \in Y
$$

The distribution over $\mathrm{X}$ can be obtained by summing out all the other variables from any joint distribution containing $\mathrm{X}$. We can use the conditional probabilities instead of joint probabilities to compute the probabilities over $\mathrm{X}$ as shown in equation (3):

$$
P(X)=\sum_{y} P(X \mid y) . P(y) ; y \in Y
$$

Finally, the model computes the conditional probabilities $P\left(O_{i} \mid\right.$ fault, Product $)$ over node $O_{i}$ with $(i=1 \cdots n)$ given by detected fault according to the Product as illustrated in the next equation:

$$
\begin{array}{r}
P\left(O_{i} \mid \text { fault, Product }\right)=\prod_{j=1}^{n} \sum_{O_{j}} P\left(O_{i}\right) . P\left(O_{j} \mid O_{i}\right) . \\
P\left(\text { fault } \mid O_{1}, \ldots, O_{j}, \ldots, O_{n}, \text { Product }\right)
\end{array}
$$

with $j=1 \cdots n, j \neq i$.

Hence, the previous sections show that a set of possible origins is dynamically determined when a fault is detected. And when the database is available, the corresponding conditional probabilities are calculated by diagnosis model. This set of possible origins and these probability values are sent and memorized on the database by the coordination level. They are used to support decision in maintenance operations.

The advantages of proposed model are: first to locate in real time a possible fault origins set, second to reduce the space of the possible fault origins set by the evaluation of suspect level, and finally less workload for structure identification of BN model. In this model, the set of possible root causes is significantly reduced by a logical diagnosis model. Next, this is used to simplify a structural identification of BN model. Thus, the BN model receives a graphical structure with only elements at the possible origins of a detected fault. Other elements not related to the detected fault are rejected. Consequently, it does not need to compute all probabilities corresponding with all elements of system. Therefore, the probabilistic values given by $\mathrm{BN}$ model help us to continue reducing the set of possible origins. This implies that we can save the recovery time of a production system. This also shows that the combination of a deterministic approach (logical diagnosis) and a probabilistic approach (BN) help us to locate more precisely and quickly equipment at that the root causes of a detected fault. Consequently, this proposed model is appropriate to apply for fault diagnosis in a flexible manufacturing system with large space of the database and identification a solution to the more approaching the optimal model.

In this paper, we propose an example to explain the application of proposed diagnosis model in the case of a production system in Section 5.

\section{APPLICATION EXAMPLE}

In this section, an example of a production system as described in Fig.5 consists of 4 machines and one metrology. We consider that this production system is integrated with a diagnosis model which has a diagnosis mechanism as proposed in above section. A sensors system is installed to detect the quality of each machine ( good or bad) and send it to the coordination level of a control system. The product quality ( good or bad) is detected by metrology. If the quality of a product is $b a d$, it concludes that this product has a fault. In this example, we consider that the failure can propagate from one machine to another one following the product flows. Hence, we will locate the machines at the possible root causes of this detected fault.

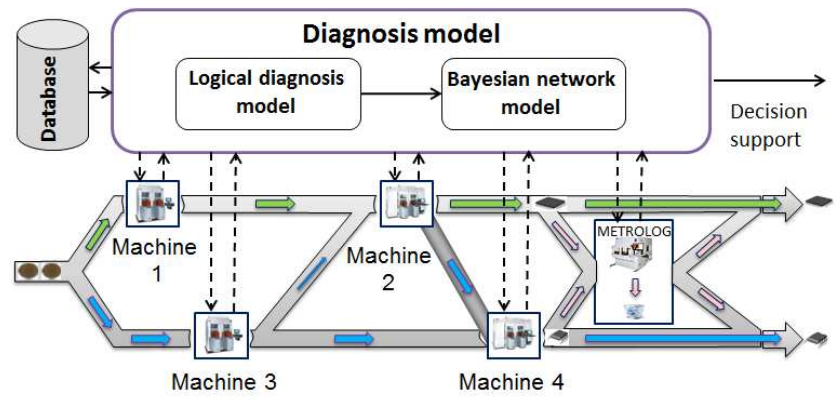

Fig. 5. A production system

The logical diagnosis model determines an evolution chain through the Operation Models of all machines. Hence, a model of machines evolutions is constructed as shown in Fig.6. The reliable elements (black nodes) are removed, while the suspect elements (white nodes) are retained based on the exploitation of sensors system. These describe a directed graphical model corresponding with the product flow. When a fault is detected at the metrology, following the back failure propagation, we obtain a set $\{M 1, M 2, M 4\}$ that are the possible fault origins. 


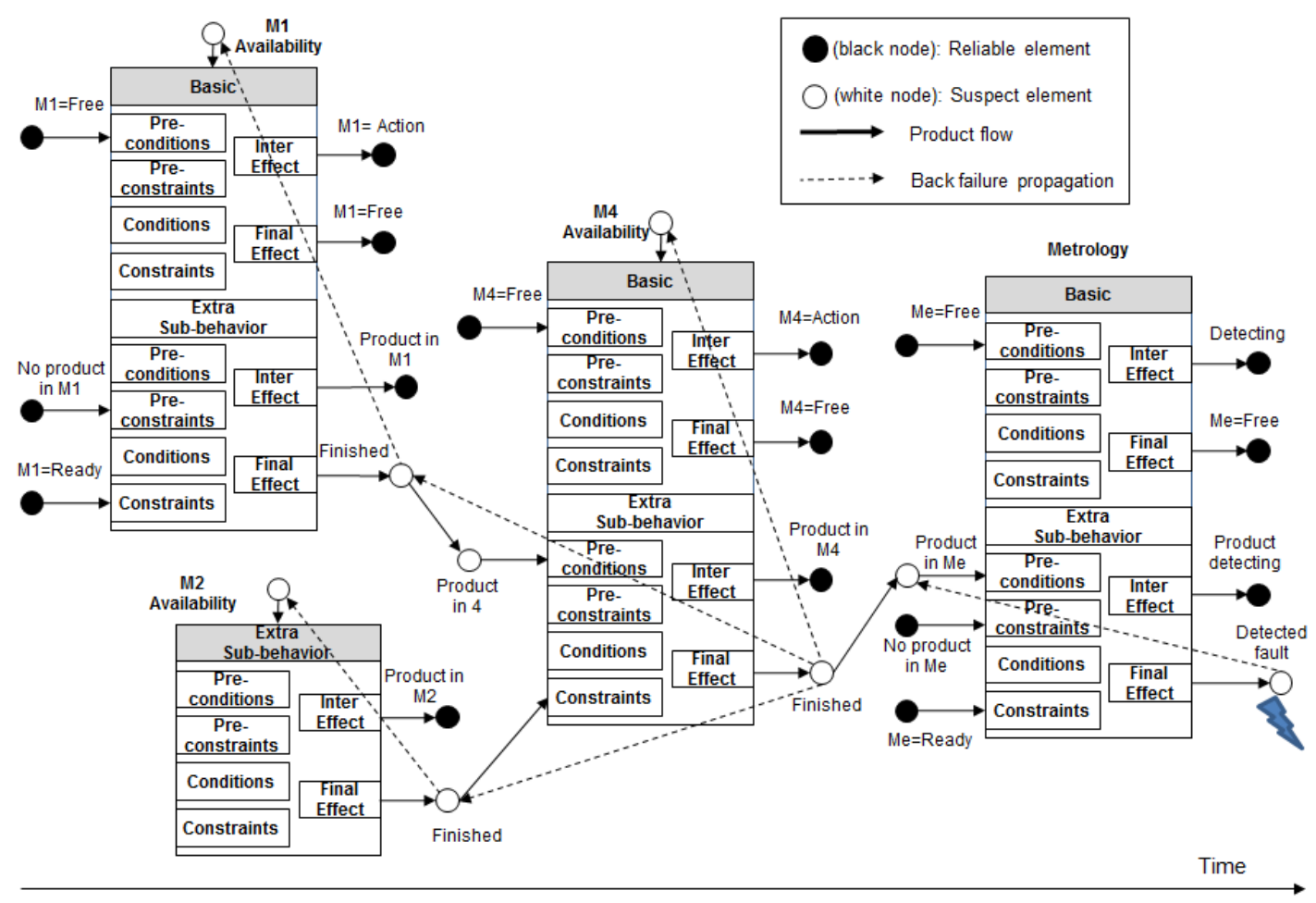

Fig. 6. Machines evolutions model following the product flows

The set $\{M 1, M 2, M 4\}$ is dynamically transformed to a graphical structure of BN model by Algorithm 1 as presented in Section 4. This graphical structure and the conditional probabilities between the parent-nodes and child-notes are illustrated in Fig.7.

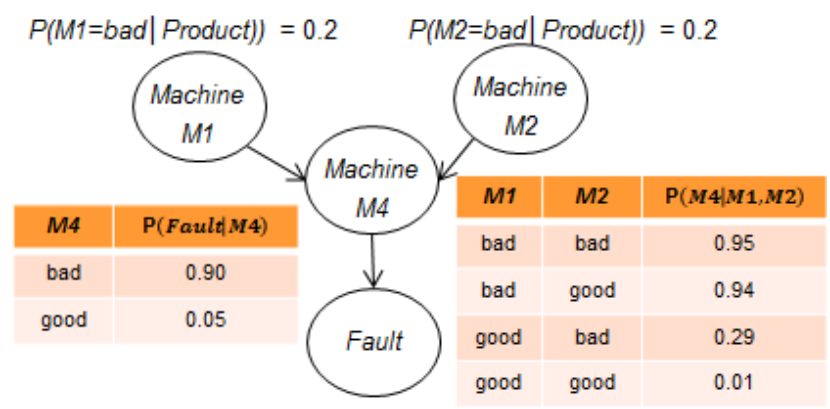

Fig. 7. The graphical structure and the conditional probabilities of its nodes

Where:

- $\quad P(M 1=$ bad $\mid$ Product $), \quad P(M 2=$ bad $\mid$ Product $)$ are the probabilities of $M 1$ and $M 2$ qualified as bad corresponding to the product.

- $\quad P(M 4 \mid M 1, M 2)$ is the conditional probability of $M 4$ in bad state given by its parents $M 1, M 2$.

- $\quad P($ fault $\mid M 4)$ is the conditional probability of product in bad state given by its parent $M 4$.

Consequently, the conditional probabilities of machines (given when product is in bad state) are computed as shown in TABLE I.
TABLE I. THE PROBABILITY RESULTS

\begin{tabular}{c||c||c}
\hline$P(M 1=$ bad $\mid$ fault $)$ & $P(M 2=$ bad $\mid$ fault $)$ & $P(M 4=$ bad $\mid$ fault $)$ \\
\hline \hline 0,08949 & 0,06764 & 0.717 \\
\hline
\end{tabular}

The result in Fig.7 shows that the possible origins of the fault detected on product by metrology may be one machine of the set $\{M 1, M 2, M 4\}$. Furthermore, the result shown in TABLE I concludes that the conditional probability $P(M 4=b a d \mid$ fault $)$ of node $M 4$ is maximum value. Consequently, the machine $M 4$ must be checked in the first place by a maintenance operator.

This example also implies that if we do not use the BN, the set of possible origins have 3 members $\{M 1, M 2, M 4\}$. Thus, we must detect all these 3 members. While if the $\mathrm{BN}$ model is used, the computed conditional probabilities helps to make decision what the member has highest suspect level. In addition, if the diagnosis process is performed based on a $\mathrm{BN}$ model without the Logical Diagnosis model, all the machines in Fig.5 may be possible origins of detected fault. We must use a learning method to identify the graphical structure of the $\mathrm{BN}$. The computation time and work-load become larger than the case of within Logical Diagnosis model.

Indeed, the combination of two techniques allows making use of its advantages and avoiding inconveniences. The proposed model is dedicated to advance in real time diagnosis and simplify the decision making for a maintenance strategy. It is feasible to apply on the flexible manufacturing systems under dynamical production environment. 


\section{CONCLUSION}

A diagnosis model for accurate fault diagnosis and corrective maintenance in a complex manufacturing system has been proposed. This model is based on a logical diagnosis approach which can limit a part of equipment at the possible origins of detected fault. The logical diagnosis approach is extended with a BN model to evaluate the suspect level of each member of a set of possible fault origins. Besides, the logical diagnosis also helps to simplify the failure model identification in the learning phase of $\mathrm{BN}$.

The proposed diagnosis model is appropriate in a complex manufacturing environment which has the characteristics such as: multiple products, multiple lines, multiple recipes, multiple human factors. The elements of system often change under flexible production activities. The proposed model has the advantage of dynamic localization of potential root causes, in less time and less workload is needed to compute the conditional probability values.

This study is first based on precisely and quickly locating the faults which can come from equipment and products. Moreover, the approach must take into account the different fault sources as recipes and human factors. Consequently, our future work is focused on the fault diagnosis of a general manufacturing system with multiple-fault sources.

\section{REFERENCES}

[1] E. Chanthery and Y. Pencole. Monitoring and active diagnosis for discrete-event systems, In 7th IFAC symposium on fault detection, supervision and safety of technical systems. , Barcelona, Spain, pp. 15451550, 2009.

[2] E. Deschamps and E. Zamai. Diagnosis for control system reconfiguration, In IFAC Management and Control of Production and Logistics, volume 4, no.1, pp. 377-382, 2007.

[3] S. Verron, T. Tiplica, and A. Kobi. Fault diagnosis with bayesian networks: Application to the tennessee eastman process, in Industrial Technology, ICIT, IEEE, pp. 98-103, 2006.

[4] P. Weber, G. Medina-Oliva, C. Simon and B. Iung. Overview on Bayesian networks applications for dependability, risk analysis and maintenance areas, Engineering Applications of Artificial Intelligence, volume 25, issue 4, pp. 671-682, 2012.

[5] M.F. Bouaziz, E. Zamai, F. Duvivier and S. Hubac. Dependability of complex semiconductor systems: Learning Bayesian networks for decision support, 3rd International Workshop on Dependable Control of Discrete Systems (DCDS), pp. 7-12, 2011.

[6] A. A. Freitas. Data mining and knowledge discovery with evolutionary algorithms, Springer, 2002.

[7] S. Brown. Strategic manufacturing for competitive advantage: transforming operations from shop floor to strategy, Prentice Hall, 1996.

[8] E. J. Phillips. Manufacturing Plant Layout: Fundamentals and fine points of optimum facility design, Society of Manufacturing Engineers Dearborn, MI, 1997.

[9] C. T. Papadopoulos, M. E. OKelly, M. J. Vidalis, and D. Spinellis. Analysis and design of discrete part production lines, vol. 31. Springer, 2009.

[10] A. Patterson Hine, G. Aaseng, G. Biswas, S. Narasimhan, and K. Pattipati. A review of diagnostic techniques for ISHM applications, 2005.

[11] A. Jones and A. Saleh. A multi-leve/multi-layer architecture for intelligent shopfloor control, International Journal of Computer Integrated Manufacturing, volume 3, issue 1, pp. 60-70, 1990.

[12] S. Henry, E. Zamai, and M. Jacomino.Controlled system model adapted for control law synthesis, 16th IFAC World Congress (IFAC05), Prague, Czech Republic, 2005.
[13] J. White, D. Benavides, D.C. Schmidt, P. Trinidad, B. Dougherty and A. Ruiz-Cortes. Automated diagnosis of feature model configurations, Journal of Systems and Software, volume 83, issue 7, 1094-1107, 2010.

[14] K. Durga Rao, V. Gopika, V. V. S. Sanyasi Rao, H. S. Kushwaha, A. K. Verma, and A. Srividya. Dynamic fault tree analysis using Monte Carlo simulation in probabilistic safety assessment, Reliab. Eng. Syst. Saf., vol. 94, no. 4, pp. 872-883, Apr. 2009.

[15] Abdelwahed, Sherif, Gabor Karsai, and Gautam Biswas. System Diagnosis using Hybrid Failure Propagation Graphs, in The 15th International Workshop on Principles of Diagnosis, Carcassonne, France, 2004.

[16] S. Strasser and J. Sheppard. Diagnostic alarm sequence maturation in timed failure propagation graphs, In AUTOTESTCON, IEEE, pp. 158$165,2011$.

[17] S. Khomfoi and L. M. Tolbert. Fault Diagnostic System for a Multilevel Inverter Using a Neural Network, IEEE Trans. Power Electron, vol. 22, pp. 1062-1069, 2007.

[18] Q.B. Duong, E. Zamai, K.Q. Tran-Dinh. Confidence estimation of feedback information for logicdiagnosis, Engineering Applications of Artificial Intelligence, volume 26, issue 3, pp. 1149-1161, 2013.

[19] R. M. Neal and G. E. Hinton. A view of the EM algorithm that justifies incremental, sparse, and other variants, In Learning in graphical models, Springer, pp. 355-368, 1998

[20] E. Zamai, A. Chaillet-Subias and M. Combacau. An architecture for control and monitoring of discrete events systems, Computers in Industry, volume 36, issues 1-2, pp. 95-100, 1998.

[21] C. E. Manfredotti. Modeling and inference with relational dynamic Bayesian networks, Advances in artificial intelligence, Springer, 2009. 